\title{
Xylitol inhibits in vitro and in vivo angiogenesis by suppressing the NF-kB and Akt signaling pathways
}

\author{
EUI-YEUN YI ${ }^{1,2}$ and YUNG-JIN KIM ${ }^{1}$ \\ ${ }^{1}$ Department of Molecular Biology, College of Natural Science, ${ }^{2}$ Research Institute for Genetic Engineering, \\ Pusan National University, Busan 609-735, Republic of Korea
}

Received January 23, 2013; Accepted March 26, 2013

DOI: 10.3892/ijo.2013.1917

\begin{abstract}
Angiogenesis is an important process involved in tumor growth and metastasis. Many studies have investigated the use of natural compounds such as angiogenic inhibitors. Xylitol is a 5-carbon sugar alcohol and is an artificial sweetener that has been used in chewing gums to prevent tooth decay. Xylitol has been also known to inhibit inflammatory cytokine expression induced by lipopolysaccharide (LPS). Since angiogenesis and inflammation share a common signaling pathway, we investigated the role of xylitol in angiogenesis. Xylitol inhibited the migration, invasion and tube formation of human umbilical vein endothelial cells (HUVECs). Xylitol also inhibited in vivo angiogenesis in a mouse Matrigel plug assay. Furthermore, mRNA expression of vascular endothelial growth factor (VEGF), VEGFR-II (KDR), basic fibroblast growth factor (bFGF), bFGFR-II, matrix metalloproteinase-2 (MMP-2) and MMP-9 of HUVECs decreased following treatment with xylitol. These anti-angiogenic effects of xylitol are exerted through inhibition of NF- $\kappa \mathrm{B}$ and Akt activation. Taken together, these results suggest that xylitol acts as a beneficial angiogenesis inhibitor.
\end{abstract}

\section{Introduction}

Angiogenesis is the development of new capillaries from preexisting vasculature during tumor growth and metastasis $(1,2)$ and is regulated by the balanced action of angiogenic activators and inhibitors $(3,4)$. This process is complex and involves diverse cellular actions, such as degradation of the extracellular matrix (ECM), proliferation and migration of endothelial cells, and morphological differentiation of endothelial cells to form tubes (5).

Angiogenesis and inflammation are important processes involved in tumor growth and expansion (6-8). Natural compounds with anti-inflammatory properties can also have

Correspondence to: Professor Yung-Jin Kim, Department of Molecular Biology, College of Natural Science, Pusan National University, Busan 609-735, Republic of Korea

E-mail: yjinkim@pusan.ac.kr

Key words: xylitol, angiogenesis, $\mathrm{NF}-\kappa \mathrm{B}, \mathrm{Akt}$, anticancer anti-angiogenic activities since these processes share a common signaling pathway such as $\mathrm{NF}-\kappa \mathrm{B}$ and Akt $(9,10)$. One of these natural compounds is xylitol. Xylitol, first derived from Birch trees in Finland in the 20th century (11), is a 5-carbon sugar alcohol (12) (Fig. 1). It has been widely used as a sugar substitute and is found in the fibers of many fruits and vegetables (13). Xylitol can also be used as an artificial sweetener in chewing gums to prevent tooth decay $(14,15)$.

In a recent study, xylitol was shown to inhibit lipopolysaccharide (LPS)-induced inflammatory cytokine expression (16). Because inflammation and angiogenesis share a common signaling pathway $(17,18)$, we tested whether xylitol has anti-angiogenic effects by using in vitro and in vivo angiogenesis assays. We found that xylitol suppressed tube formation, migration and invasion of human umbilical vein endothelial cells (HUVECs). Xylitol also inhibited in vivo angiogenesis in a Matrigel plug assay. Additionally, xylitol downregulated the mRNA expression of vascular endothelial growth factor (VEGF), VEGFR-II (KDR), basic fibroblast growth factor (bFGF), bFGFR-II, matrix metalloproteinase-2 (MMP-2) and MMP-9 of HUVECs. We also found that xylitol suppressed NF- $\kappa \mathrm{B}$ and Akt activation in HUVECs.

\section{Materials and methods}

Materials and reagents. HUVECs were purchased from InnoPharmaScreen (Chungnam, Korea). Matrigel was obtained from Collaborative Biomedical Products (Bedford, MA, USA) for the mouse Matrigel plug assay. Basic fibroblast growth factor (bFGF) and heparin were obtained from PeproTech (Gaithersburg, MD, USA). Fetal bovine serum (FBS), penicillin and streptomycin were purchased from JBI (Daegu, Korea). Drabkin reagent kit 525 was purchased from Sigma (St. Louis, MO, USA). The $8-\mu \mathrm{m}$ pore Transwell filter chambers were purchased from Corning-Costar (Corning, NY, USA). Antibodies for NF- $\kappa \mathrm{B}$, phosphor-NF- $\kappa \mathrm{B}, \mathrm{Akt}$, and phosphor-Akt were purchased from Cell Signaling Technology Inc. (Danvers, MA, USA). Xylitol and gelatin were purchased from Sigma.

HUVECs. HUVECs were grown in M199 supplemented with heat-inactivated $20 \%$ fetal bovine serum (JBI), $20 \mathrm{ng} / \mathrm{ml}$ of $\mathrm{bFGF}, 10 \mathrm{U} / \mathrm{ml}$ of heparin, $100 \mathrm{U} / \mathrm{ml}$ of penicillin and $100 \mu \mathrm{g} / \mathrm{ml}$ of streptomycin in a $37^{\circ} \mathrm{C}$ incubator with a humidified atmosphere containing $5 \% \mathrm{CO}_{2}$. 


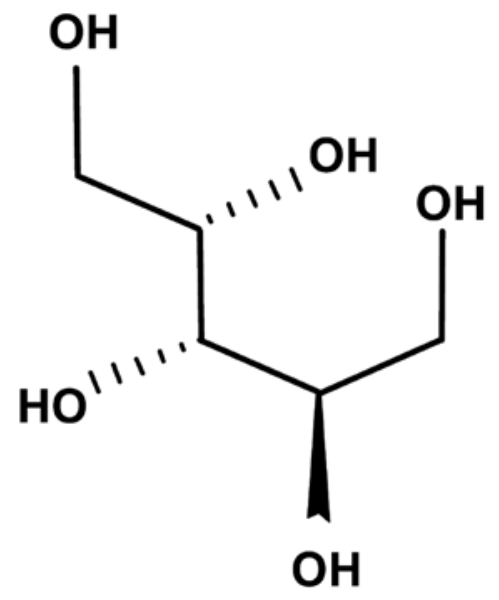

Figure 1. Structure of xylitol.

Animals. Seven-week-old, specific pathogen-free (SPF) male C57BL/6 mice were supplied by Hyochang Science and Samtako (Daegu and Kyung-gi, Korea). They were provided with autoclaved tap water and lab chow ad libitum and were housed at $23 \pm 0.5^{\circ} \mathrm{C}, 10 \%$ humidity under a 12 -h light-dark cycle. The animal protocol used in this study was reviewed on the ethical procedures and scientific care, and approved by the Pusan National University-Institutional Animal Care and Use Committee (PNU-IACUC).

In vitro tube formation assay. HUVECs $\left(2 \times 10^{4}\right.$ cells $)$ were seeded on a layer of previously polymerized Matrigel and treated with or without xylitol. Matrigel culture was incubated at $37^{\circ} \mathrm{C}$. After $6 \mathrm{~h}$, changes in cell morphology were captured through a phase contrast microscope and photographed at $\times 40$ magnification. Each sample was assayed in duplicate and independent experiments were repeated three times.

In vitro wounding migration assay. HUVECs were seeded onto 24-well culture plates until confluence and left overnight. Media was aspirated the next day, and cells were scratched with a $200 \mu \mathrm{l}$ pipette tip along the diameter of the well. Cells were washed twice with PBS and incubated at $37^{\circ} \mathrm{C}$ and $5 \% \mathrm{CO}_{2}$. After wounding, the cultures were washed with serum-free medium and further incubated in M199 with $1 \%$ serum, $1 \mathrm{mM}$ thymidine and/or xylitol. These culture conditions minimized proliferation of HUVECs. Wound diameters were photographed at 18 to $24 \mathrm{~h}$. Wound closure was determined by measurement with optical microscopy at $\mathrm{x} 40$ magnification. Migration was quantitated by counting the number of cells that moved beyond the reference line. Each sample was assayed in duplicate, and independent experiments were repeated three times.

In vitro invasion assay. Invasiveness of HUVECs was performed in vitro using a Transwell chambers system (Corning-Costar) with $8.0-\mu \mathrm{m}$ pore polycarbonate filter inserts. The upper side was coated with $10 \mu \mathrm{l}$ of Matrigel $(0.5 \mathrm{mg} / \mathrm{ml})$ at room temperature for $1 \mathrm{~h}$. Cells $\left(2 \times 10^{4}\right.$ cells) and xylitol in serum-free medium was placed in the upper part of the filters, and full medium was treated in the lower parts. Cells were incubated at $37^{\circ} \mathrm{C}$ for $24 \mathrm{~h}$, fixed with methanol, and then stained with hematoxylin and eosin. Cells on the upper surface of the membrane were removed by wiping with a cotton swab. Cell invasion was determined by counting whole cell numbers in a single filter by optical microscopy at $\mathrm{x} 40$ magnification. Each sample was assayed in duplicate and independent experiments were repeated three times.

In vivo mouse Matrigel plug assay. C57BL/6 mice (7 weeks of age) were injected subcutaneously into $500 \mu \mathrm{l}$ of Matrigel (Collaborative Biomedical Products, Bedford, MA, USA) containing bFGF $(100 \mathrm{ng} / \mathrm{ml})$ and heparin $(50 \mathrm{U} / \mathrm{ml})$ without or with xylitol. After injection, the Matrigel rapidly formed a plug. After 7 days, skin of the mouse was pulled back to expose the Matrigel plug, which remained intact. After quantitative differences were noted and photographed, hemoglobin content was measured using the Drabkin reagent kit 525 (Sigma) for quantification of blood vessel formation. The amount of hemoglobin was calculated from a known amount of hemoglobin assayed in parallel. Independent experiments were repeated twice and at least five mice in each experiment were used.

Reverse transcription-polymerase chain reaction (RT-PCR). Total RNA from HUVECs was isolated using TRIzol reagent (Invitrogen Corporation, Carlsbad, CA, USA) according to the manufacturer's instructions. First-stranded cDNA was synthesized by M-MLV reverse transcriptase (Promega Corporation, Madison, WI, USA) with $2 \mu \mathrm{g}$ each of DNA-free total RNA sample and oligo (dT)15 (Life Technologies, Grand Island, NY, USA) according to the manufacturer's instructions. Equal amounts of cDNA were subsequently amplified by PCR in a $20 \mu \mathrm{l}$ reaction volume containing $1 \mathrm{X}$ PCR buffer, dNTP mixture, $10 \mu \mathrm{M}$ of each specific primer and $\mathrm{i}-\mathrm{Taq}^{\mathrm{TM}}$ DNA polymerase (iNtRON Biotechnology, Sungnam, Korea). Amplification products were electrophoresed on $1 \%$ agarose gels and visualized by GelRed (Biotium Inc., Hayward, CA, USA) staining under ultraviolet trans-illumination.

Western blot analysis. HUVECs were treated with or without xylitol for $24 \mathrm{~h}$ in medium. Total cell lysates were prepared by addition of PRO-PREP Protein Extraction Solution (iNtRON Biotechnology), including $1 \mathrm{mM}$ sodium orthovanadate. Equal amounts $(30 \mu \mathrm{g})$ of samples were resolved by electrophoresis on a $10 \%$ SDS-polyacrylamide gel, transferred to a membrane and sequentially probed with antibody. The following primary antibodies were used at the indicated dilutions: total NF- $\mathrm{kB}$ and anti-phospho-NF-kB, total Akt and anti-phospho-Akt, 1:1,000 in 5\% BSA in TBS-T.

\section{Results}

Xylitol inhibits vascular network formation in HUVECs. Because differentiation of endothelial cells into a capillary-like network is important for the process of angiogenesis, we tested the effect of xylitol on the morphological differentiation of endothelial cells in vitro. HUVECs were placed on a Matrigel-coated plate and incubated. Endothelial cells formed weak capillaries on Matrigel beds, and these tubes became stronger and more robust with elongated networks over 6-24 h. HUVECs on Matrigel formed a blood vessel-like network in the absence of xylitol (Fig. 2), whereas treatment with xylitol for $18 \mathrm{~h}$ resulted in broken, shortened and narrow tube 


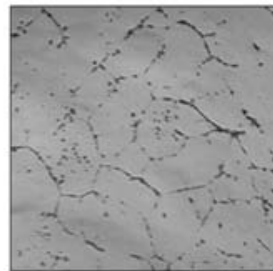

Control

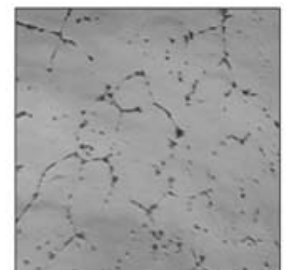

Xylitol $0.1 \mathrm{mM}$

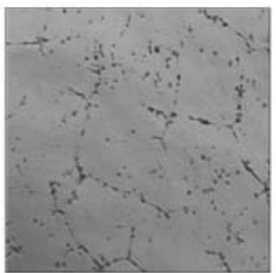

Xylitol $0.3 \mathrm{mM}$

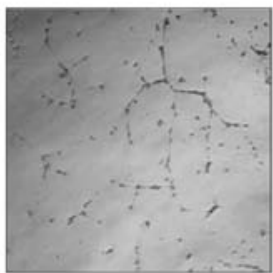

Xylitol $0.5 \mathrm{mM}$

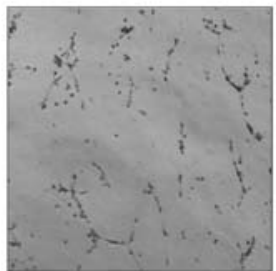

Xylitol $1 \mathrm{mM}$
Figure 2. Xylitol inhibits tube formation of HUVECs. The effect of xylitol on tube formation of HUVECs was examined using an in vitro tube formation assay. Indicated amounts $(0.1,0.3,0.5$ and $1 \mathrm{mM})$ of xylitol were added and the cells were incubated for $18 \mathrm{~h}$. Changes in cell morphology were observed using a phase contrast microscope (x40) and photographed. Representative photographs of tube formation of endothelial cells cultured on polymerized Matrige layers revealed the inhibitory effect of xylitol on the formation of capillary-like structures. This experiment was repeated three times.

$\mathbf{A}$
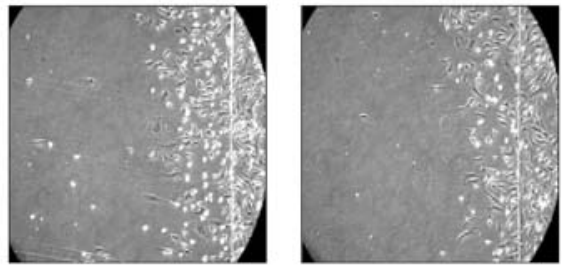

Control

Xylitol 0.1 mM

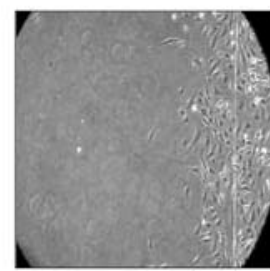

Xylitol $0.3 \mathrm{mM}$

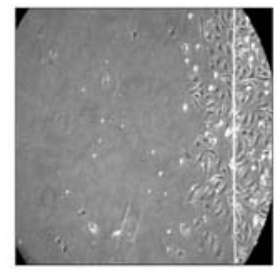

Xylitol $0.5 \mathrm{mM}$

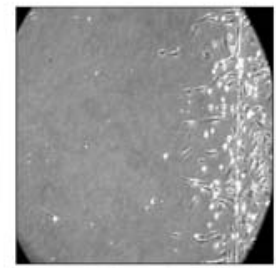

Xylitol 1 mM
B

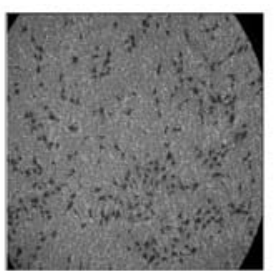

Control

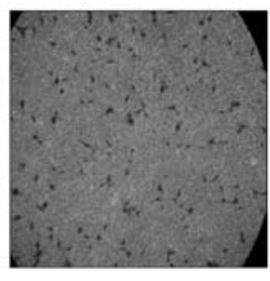

Xylitol 0.1 mM

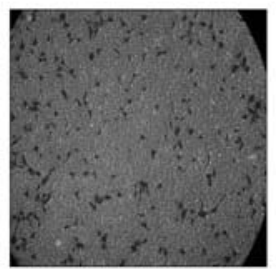

Xylitol 0.3 mM

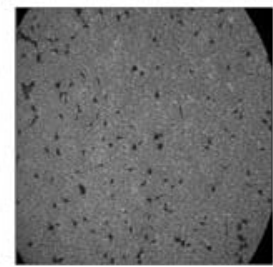

Xylitol $0.5 \mathrm{mM}$

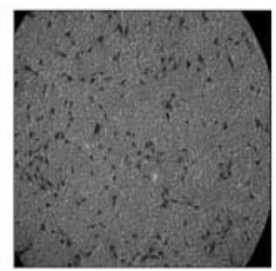

Xylitol 1 mM
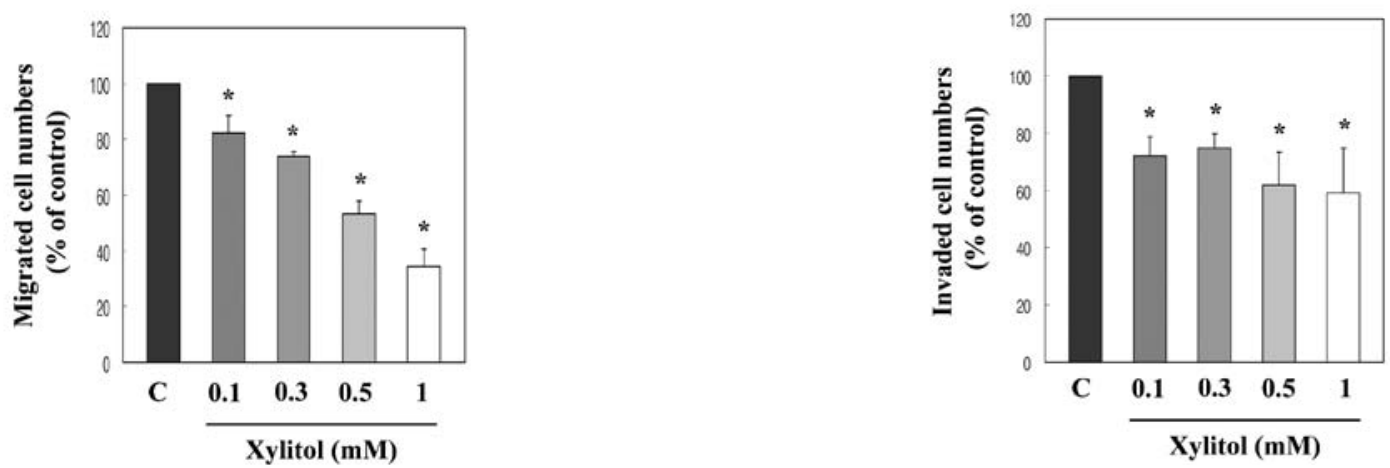

Figure 3. Xylitol inhibits HUVEC migration and invasion. (A) Migration ability of HUVECs was measured using a wound migration assay. (B) Invasion capacity was examined using the Transwell system in which the wells were coated with Matrigel. Data are mean \pm SD of three independent experiments performed in triplicate. ${ }^{*} \mathrm{p}<0.05$ vs. control. 
A
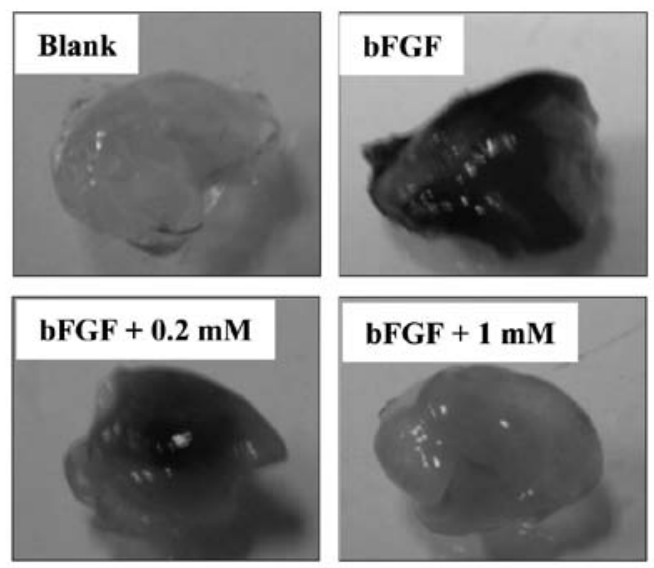

B

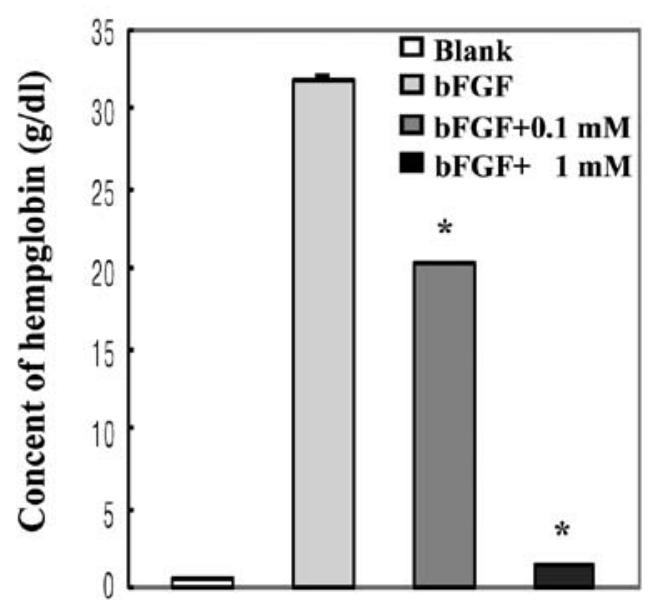

Figure 4. Xylitol suppressed in vivo angiogenesis in mouse Matrigel plug assay. (A) Matrigel plugs were photographed (x40). (B) Quantification of hemoglobin content. Each value represents the mean \pm SD of at least five animals, and the experiment was performed twice. Blank, Matrigel alone. " $\mathrm{p}<0.05$ vs. hemoglobin content of bFGF-induced implants.

observed in the Matrigel alone (blank). Matrigel plugs containing xylitol produced fewer vessels compared with plugs containing bFGF, indicating that xylitol inhibits formation of bFGF-induced neo-microvessels. We then measured the hemoglobin content inside the Matrigel plugs to quantify the anti-angiogenic effect of xylitol. The amount of hemoglobin indicates the degree of formation of a functional vasculature inside the Matrigel. The hemoglobin content of bFGF-treated plugs was $31.8 \mathrm{~g} / \mathrm{dl}$, whereas that of $1 \mathrm{mM}$ xylitol-treated plugs was profoundly lowered to approximately $1.4 \mathrm{~g} / \mathrm{dl}$ (Fig. 4B). These results indicate that xylitol has strong anti-angiogenic activity in vivo.

Xylitol downregulates mRNA expression of angiogenesisrelated genes. To determine which molecules are involved in the anti-angiogenic activity of xylitol, we examined mRNA expression of angiogenic factors and their receptors in HUVECs following xylitol treatment by using RT-PCR. As shown in Fig. 5A, VEGF mRNA expression was significantly reduced in the presence of xylitol. VEGFR-II (KDR) mRNA expression was also downregulated following treatment with xylitol in a dose-dependent manner. mRNA expression of other angiogenic molecules, including bFGF and bFGR-II, was remarkably reduced by xylitol (Fig. 5B).
A
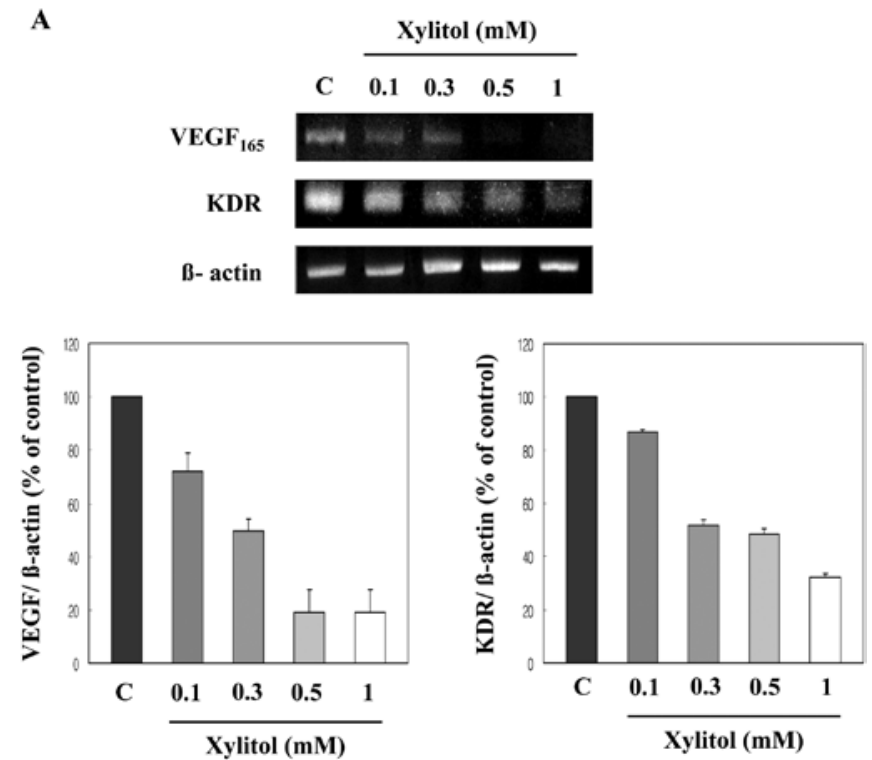

B
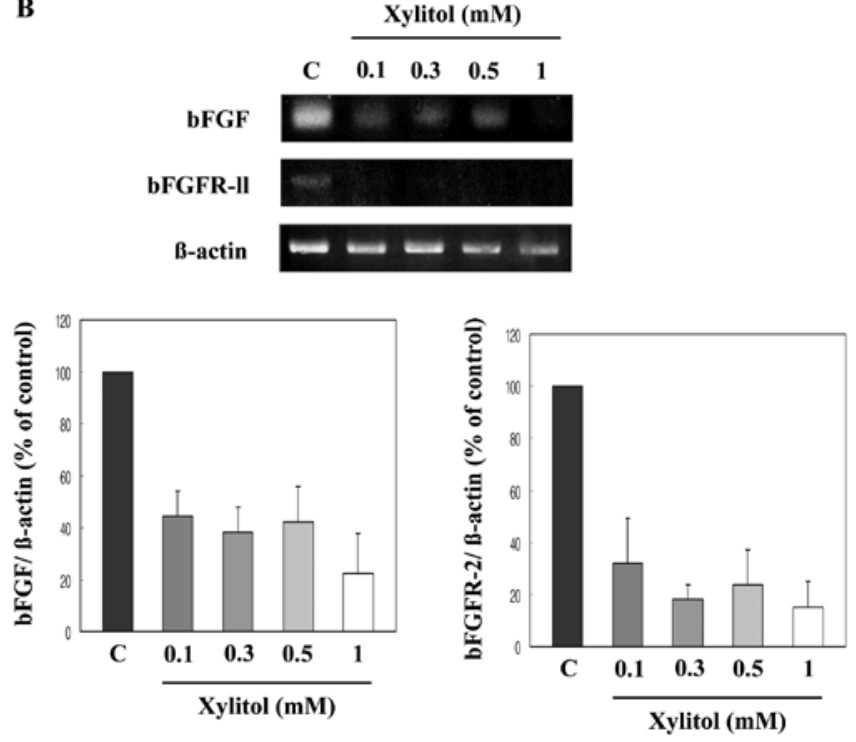

C
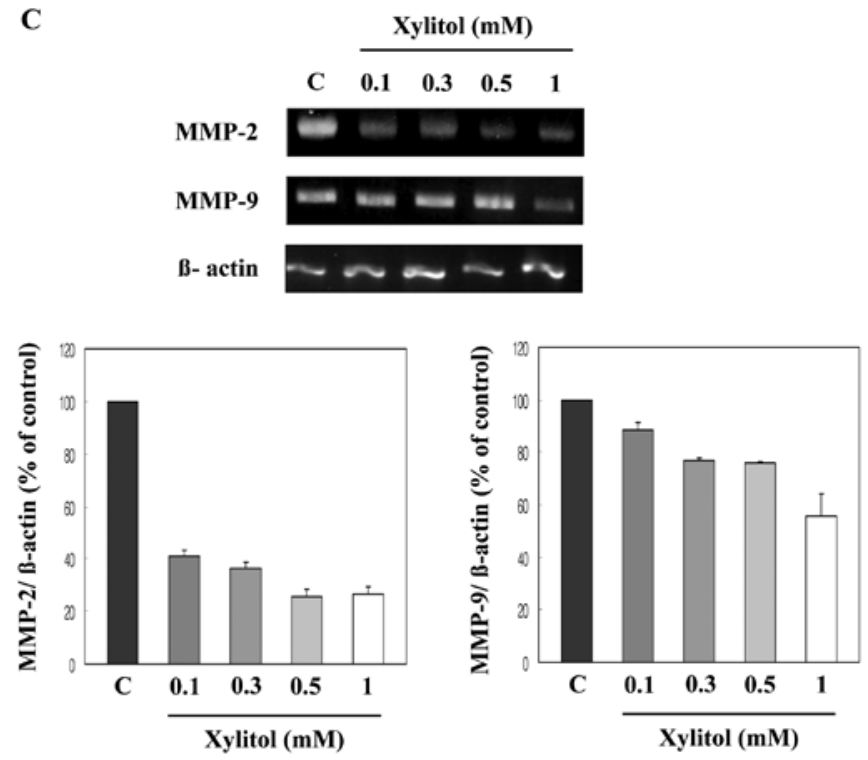

Figure 5. Xylitol downregulated angiogenesis-related gene expression. mRNA expression in xylitol-treated HUVECs was analyzed using RT-PCR. (A) VEGF and KDR, (B) bFGF and bFGFR-II, (C) MMP-2 and MMP-9. Amplification of $\beta$-actin demonstrates comparable RNA amount and quantity among samples. 


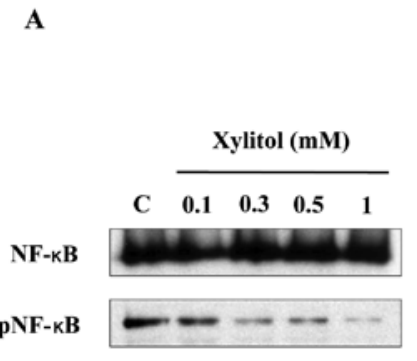

B
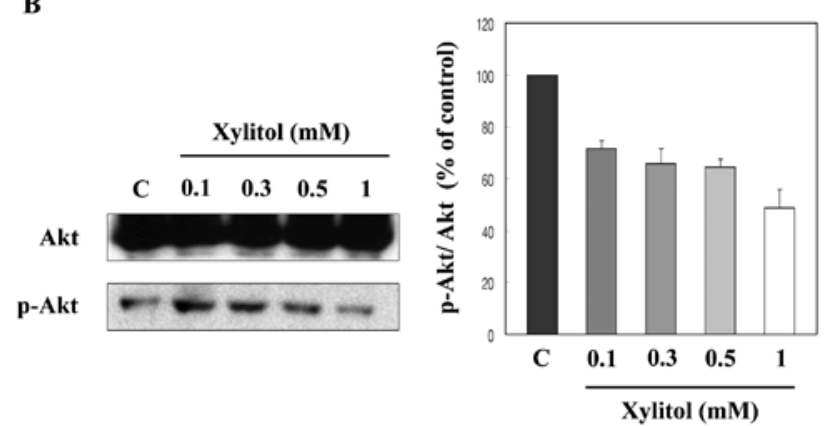

Figure 6. Downregulation of NF- $\mathrm{kB}$ and Akt activation by xylitol in HUVECs. HUVECs were treated with the indicated amounts of xylitol for $24 \mathrm{~h}$. Total proteins were prepared and analyzed by western blot analysis for each primary

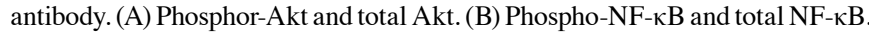
Each value was normalized to the total form and $\alpha$-tubulin.

Regulation of extracellular proteolytic activity is important for endothelial cell migration and invasion, and MMP-2 and MMP-9 are major functional molecules involved in the degradation of the extracellular membrane (21). Thus, we examined the effect of xylitol on the mRNA expression of MMP-2 and MMP-9. Expression of both molecules was markedly downregulated following treatment with xylitol in a dose-dependent manner (Fig. 5C). These data suggest that downregulation of VEGF, KDR, bFGF, bFGFR-II, MMP-2 and MMP-9 mRNA expression may be responsible for the anti-angiogenic action observed in HUVECs treated with xylitol.

Xylitol suppresses NF- $\kappa B$ and Akt activation of HUVECs. NF- $\kappa \mathrm{B}$ and Akt are the main signaling pathways involved in tumor progression and angiogenesis $(22,23)$. Therefore, we examined the effect of xylitol on NF- $\mathrm{KB}$ and Akt activation in HUVECs. In this experiment, total proteins were prepared to detect NF- $\kappa B$ and Akt phosphorylation and then analyzed by western blot analysis. As shown in Fig. 6A, NF- $\kappa$ B phosphorylation was suppressed by xylitol in a dose-dependent manner. Because NF- $\kappa B$ activation is known to be mediated by the Akt signal pathway, we then examined Akt activation (17,24). Akt phosphorylation was inhibited in the presence of xylitol in a dose-dependent manner (Fig. 6B). These results indicate that xylitol interferes with NF- $\mathrm{kB}$ and Akt phosphorylation in HUVECs.

\section{Discussion}

Angiogenesis is required for tumor growth and expansion. Angiogenesis is the formation of new blood vessels from existing vessels; these processes involve several cascades $(19,20,25)$.
Inflammation is also known to be a central process in many cancers during tumorigenesis (26). Therefore, signal transduction of angiogenesis and inflammation can occur simultaneously (27).

Xylitol was first derived from Birch trees in Finland, and it is used as a safe sweetener for diabetic patients (11). Xylitol is obtained from natural sources, including plums, strawberries, raspberries, and rowanberries $(12,28,29)$. It is used in various food products such as chewing gum, candy and soft drinks (30). Xylitol has also been used to prevent tooth decay since it inhibits the growth of Streptococcus mutans $(31,32)$.

Recently, xylitol was reported to have an inhibitory effect on LPS-induced inflammatory cytokine expression (16). Since inflammation and angiogenesis share a common signaling pathway $(17,18)$, molecules that show anti-inflammatory activity also show anti-angiogenic activity $(8,27)$. Therefore, we examined whether xylitol has anti-angiogenic effects by using in vitro and in vivo angiogenesis assays. In this study, we found that xylitol inhibited in vitro and in vivo angiogenesis. Xylitol strongly inhibited tube formation (Fig. 2), migration (Fig. 3A), and invasion (Fig. 3B) of HUVECs. Xylitol prominently inhibited the formation of neo-microvessels in the Matrigel assay (Fig. 4A), and reduced the hemoglobin content in the Matrigel plug (Fig. 4B). These results suggest that xylitol suppresses angiogenesis both in vitro and in vivo.

Tumors produce various angiogenic molecules during the angiogenesis process. VEGF, bFGF and their receptors are well known as the main stimuli for angiogenesis (33-35). Therefore, we examined the involvement of xylitol in the expression of major angiogenic factors and their receptors. Xylitol decreased the mRNA expression of key angiogenic molecules and their receptors (VEGF, KDR, bFGF and bFGFR-II) in a dose-dependent manner (Fig. 5).

MMPs are secreted as proenzymes and they regulate extracellular matrix degradation. Regulation of extracellular proteolytic activity is important for cell migration and invasion (21). Thus, we tested the effect of xylitol on mRNA expression of MMP-2 and MMP-9 by using RT-PCR. As shown in Fig. 5C, xylitol decreased the mRNA expression of both MMP-2 and MMP-9.

NF- $\mathrm{kB}$ and Akt comprise a multiunit transcription factor that plays a central role in tumorigenesis and is involved in tumor cell invasion and metastasis $(24,36)$. We examined the effect of xylitol on NF- $\mathrm{KB}$ and Akt activation by western blot analysis. Protein expression of NF-kB and Akt did not change following treatment with xylitol; however, NF- $\mathrm{KB}$ and Akt phosphorylation was suppressed in a dose-dependent manner (Fig. 6).

In summary, we found that xylitol inhibited in vitro and in vivo angiogenesis. These effects of xylitol are linked with the mRNA expression of VEGF, KDR, bFGF, bFGFR-II, MMP-2 and MMP-9. Xylitol inhibited angiogenesis by inhibiting the NF- $\mathrm{kB}$ and Akt signal pathway. Furthermore, xylitol is widely used as a sugar substitute as well as an artificial sweetener in chewing gums to prevent tooth decay. Therefore, xylitol may be a promising candidate as an inhibitor of angiogenesisrelated diseases.

\section{Acknowledgements}

This study was financially supported by the 2012 Postdoc. Development Program of Pusan National University and was 
supported by the Basic Science Research Program through the National Research Foundation of Korea (NRF) funded by the Ministry of Education, Science and Technology (2011-0024330).

\section{References}

1. Folkman J and Shing Y: Angiogenesis. J Biol Chem 267: 10931-10934, 1992.

2. Siddiqui FA, Desai H, Siddiqui TF and Francis JL: Hemoglobin induces the expression and secretion of vascular endothelial growth factor from human malignant cells. Hematol J 3 : 264-270, 2002.

3. Carmeliet P and Jain RK: Angiogenesis in cancer and other diseases. Nature 407: 249-257, 2000.

4. Ferrara $\mathrm{N}$ and Kerbel RS: Angiogenesis as therapeutic target. Nature 438: 967-974, 2005.

5. Isner JM and Asahara T: Angiogenesis and vasculogenesis as therapeutics strategies for postnatal neovascularization. J Clin Invest 103: 1231-1236, 1999.

6. Folkman J: Tumor angiogenesis; therapeutic implications. N Engl J Med 285: 1182-1186, 1971.

7. Szekanecz Z and Koch AE: Endothelial cells in inflammation and angiogenesis. Curr Drug Targets Inflam Allergy 4: 319-323, 2005.

8. Ono M: Molecular links between tumor angiogenesis and inflammation: inflammatory stimuli of macrophages and cancer cells as targets for therapeutic strategy. Cancer Sci 99: 1501-1506, 2008.

9. Karin M and Greteo FR: NF- $\kappa B$ : Linking inflammation and immunity to cancer development and progression. Nat Rev Immunol 5: 749-759, 2005.

10. Albini A, Tosetti F, Benelli R and Noonan DM: Tumor inflammatory angiogenesis and its chemoprevention. Cancer Res 65: 10639-10641, 2005.

11. Tanzer JM: Xylitol chewing gum and dental caries. Int Dent $\mathrm{J}$ 45: 65-76, 1995.

12. Bradshaw DJ and Marsh PD: Effect of sugar alcohols on the composition and metabolism of a mixed culture of oral bacteria grown in a chemostat. Caries Res 28: 251-256, 1994.

13. Makinen KK and Soderling E: A quantitative study of mannitol, sorbitol, xylitol, and xylose in wild berries and commercial fruits. J Food Sci 45: 367-371, 1980.

14. Makinen KK, Bennett CA, Hujoel PP, et al: Xylitol chewing gums and caries rates: a 40-month cohort study. J Dent Res 74: 1904-1913, 1995.

15. Söderling E, Mäkinen KK, Chen CY, et al: Effect of sorbitol, xylitol, and xylitol/sorbitol chewing gums on dental plaque. Caries Res 23: 378-384, 1989.

16. Han SJ, Jeong SY, Nam YJ, et al: Xylitol inhibits inflammatory cytokine expression induced by lipopolysaccharide from Porphyromonas gingivalis. Clin Diagn Lab Immunol 12: 1285-1291, 2005.

17. Bisacchi D, Benelli R, Venzetto C, et al: Anti-angiognesis and angioprevention: mechanisms, problems and perspectives. Cancer Detect Prev 27: 229-238, 2003.
18. Yoshimura A: Signal transduction of inflammatory cytokines and tumor development. Cancer Sci 97: 439-447, 2006.

19. Pang R and Poon RT: Angiogenesis and antiangiogenic therapy in hepatocellular carcinoma. Cancer Lett 242: 151-167, 2006.

20. Femke H and Arjan WG: Tumor vascularization: sprouting angiogenesis and beyond. Cancer Metastasis Rev 26: 489-502, 2007.

21. Bergers G, Brekken R, McMahon G, et al: Matrix matalloproteinases-9 triggers the angiogenic switch during angiogenesis. Nature Cell Biol 2: 737-744, 2000.

22. Francesco P and Antonio L: NF- $\kappa$ B solid tumors. Biochem Pharmacol: 1142-1152, 2006.

23. Lin YG, Kunnumarkkara AB, Nair A, et al: Curcumin inhibits growth and angiogenesis in ovarian carcinoma by targeting the nuclear factor- $\kappa \mathrm{B}$ pathway. Clin Cancer Res 13: 3423-3430, 2007.

24. Karin M: Nuclear factor- $\kappa \mathrm{B}$ in cancer development and progression. Nature 441: 431-436, 2006.

25. Pawel D, Kathryn H, Susan JB and Hong C: Antiangiogenic and antitumor efficacy of EphA2 receptor antagonist. Cancer Res 64: 910-919, 2004

26. Sporn MB and Suh N: Chemoprevention of cancer. Carcinogenesis 21: 525-530, 2000.

27. Jones KM, Wang H, Peskar BM, et al: Inhibition of angiogenesis by nonsteroidal anti-inflammatory drugs: insight into mechanism and implications for cancer growth ulcer healing. Nat Med 5: 1418-1423, 1999.

28. Renko M, Valkonen P, Tapiainen T, et al: Xylitol-supplemented nutrition enhances bacterial killing and prolongs survival of rats in experimental pneumococcal sepsis. BMC Microbiol 8: $1-7,2008$.

29. Georgieff M, Moldawer LL, Bistrian BR, et al: Xylitol, an energy source for intravenous nutrition after trauma. J Parenter Enteral Nutr 9: 199-209, 1985.

30. Panagiotou G, Christakopoulos P, Grotkjaer T, et al: Engineering of the redox imbalance of Fusarium oxysporum enables anaerobic growth on xylose. Metab Eng 8: 474-482, 2006.

31. Loesche WJ, Grossman NS, Earnest R, et al: The effect of chewing xylitol gum on the plaque and saliva levels of Streptococcus mutans. J Am Dent Assoc 108: 587-592, 1984.

32. Miyasawa H, Iwami Y, Mayanagi H, et al: Xylitol inhibition of anaerobic acid production by Streptococcus mutans at various pH levels. Oral Microbiol Immunol 18: 215-219, 2003.

33. Meadows KN, Bryant P and Pumiglia K: Vascular endothelial growth factor induction of the angiogenic phenotype requires ras activation. J Bio Chem 276: 49289-49298, 2001.

34. Leung DW, Cachianes G, Kuang WJ, et al: Vascular endothelial growth factor is a secreted angiogenic mitogen. Science 246: 1306-1309, 1989.

35. Asahara T, Bauters C, Zheng LP, et al: Synergistic effect of vascular endothelial growth factor and basic fibroblast growth factor on angiogenesis in vivo. Circulation 92: 11365-11371, 1995.

36. Gilmore TD: The Rel/NF- $\mathrm{B} / \mathrm{I} \kappa \mathrm{B}$ signal transduction pathway and cancer. Cancer Treat Res 115: 241-265, 2003. 